\title{
Квантовохимическое моделирование строения молекул иминодиуксусной кислоты и ее производных в водной среде
}

\author{
Даринский Б.М., Петрушенко Р.В., Селеменев В.Ф. \\ ФГБОУ ВО «Воронежский государственный университет», Воронеж
}

Поступила в редакцию 26.03.2018 г.

DOI: https://doi.org/10.17308/sorpchrom.2018.18/501

Проведены компьютерные вычисления атомного строения молекулы иминодиуксусной кислоты и ее гидратов в вакууме и водной среде. Показано, что учет диэлектрической проницаемости водной среды приводит к изменению длин ковалентных связей между атомами, валентных углов, эффективных зарядов и энергии связей. При этом топология химических связей изомера не изменяется. Присоединение одной молекулы воды приводит к смене позиции иона водорода, связанной с переносом его от иона кислорода к иону азота, что приводит к образованию цвиттер-структуры. Показано, присоединение молекулы воды к молекуле иминодиуксусной кислоты сопровождается возникновением трехцентровой ковалентной связи, что является физико-химическим механизмом термодинамической устойчивости цвиттер-структуры молекулы в водной среде.

Ключевые слова: иминодиуксусная кислота, ковалентная связь, цвиттер-структура, водная среда.

\section{Quantum chemical modeling of molecule structure of iminodiacetic acid in aqueous medium}

\author{
Darinskii B.M., Petrushenko R.V., Selemenev V.F. \\ Voronezh state University, Voronezh
}

In the present work the problem of computer modeling of atomic and electronic structure of molecule of iminodiacetic acid in vacuum and aqueous media solved. Relevance of this research direction is caused by use of this substance as initial product for synthesis of wide range of complexes and complexonat having different function and to creation of the modified surfaces of inorganic materials. The molecule of IDA is considered as tridentate complexon in which complexing ion is nitrogen anion, and electronical ligands hydrogen atom and two functional groups of $\mathrm{CH} 2 \mathrm{COOH}$.

Calculations of structures, energy and electronic wave functions were carried out by means of the Gaussian 09 and GaussView programs. As calculation method B3LYP in basis 6-311G has been used (d, p). Choice of calculation method of B3LYP/6-311G $(\mathrm{d}, \mathrm{p})$ is caused by the best consent of the experimental and calculated power characteristics of complexes of metals with organic ligands.

One of results is equilibrium and metastable configurations of molecule of IDA in vacuum. Accounting of water environment as dielectric environment has not led to change of configuration of molecule with the greatest binding energy. (in some percent) lengths of valent bonds, valence dihedral angles have undergone some changes. More considerable changes are found for effective charges. All charges have been increased on the module that has been explained to the induced polarization of the environment.

Accession of one water molecule leads to displacement of hydrogen ion from oxygen ion to nitrogen ion and to formation of zwitter-structure of the received hydrate. It is shown, accession of water molecule to molecule of iminodiacetic acid is followed by emergence of three-center covalent bond that is the physical - 
chemical mechanism of thermodynamic stability of zwitter-structure of iminodiacetic acid molecule in aqueous medium.

Keywords: iminodiacetic acid, covalent bond, zwitter-structure, aqueous medium.

\section{Введение}

Иминодиуксусная кислота (ИДА) примерно с середины двадцатого века и до настоящего времени служит важным исходным продуктом для синтезов широкого круга комплексов и комплексонатов различного назначения. Применение этих продуктов нашло в ряде различных областей науки и практики. В аналитической химии широкое распространение получил метод титриметрического анализа, ставший основой для создания и развития «хелатометрии» [1]. Комплексы используются в хроматографическом и других физико-химических методах анализа состава вещества [2], в медицине [3-5], очистке воды, рекультивации почв и многих других областях науки и техники [6]. Особо отметим многообещающее направление в химии, основанное на использовании и исследовании модифицированных поверхностей неорганических материалов [7-14]. Огромное разнообразие элементных составов и структур поверхностей а также их модификаторов, технических возможностей реализации нужных модифицированных структур создает широчайшие возможности для открытия новых эффектов и решения различных задач [15]. Несмотря на довольно раннее экспериментальное исследование химических свойств указанных комплексов, начатых работами Шварценбаха [16-18], до настоящего времени они не стали объектом компьютерного исследования.

В настоящей работе ставится задача компьютерного моделирования атомного и электронного строения молекулы ИДА.

\section{Теоретическая часть}

Строение молекулы ИДА. Молекула ИДА рассматривается как тридентатный комплексон, в котором комплексообразующим ионом является анион азота, а электродонорными лигандами атом водорода и две функциональные группы $\mathrm{CH}_{2} \mathrm{COOH}$. Экспериментально установлено, что в результате кристаллизации из водных растворов молекула ИДА может иметь три различных модификации.

Для молекулы и всех ионов ИДА в водных растворах типично бетаиновое строение и цвиттер-ионная структура. Последовательность смены ионных состояний ИДА при уменьшении кислотности водного раствора представлена на рисунке 1 $[19,20]$.

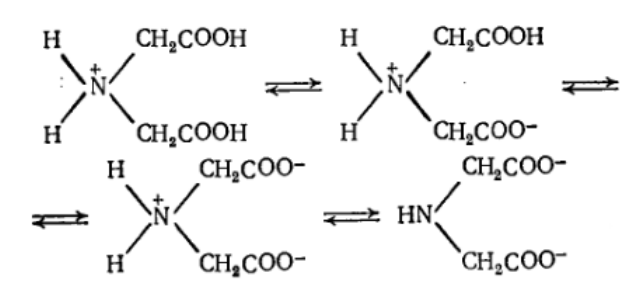

Рис.1. Последовательность диссоциации молекулы ИДА в водных растворах с увеличением основности [19].

Отмечается, что молекула ИДА имеет гибкую структуру и может иметь несколько изомеров. При кристаллизации из водных растворов обнаружено три изомера, имеющих цвиттер-структуру [21]. 


\section{Эксперимент}

Вычисления структур и энергий проводились при помощи программ Gaussian 09 и GaussView. В качестве расчетного метода был применен B3LYP в базисе 6$311 \mathrm{G}(\mathrm{d}, \mathrm{p})$. Выбор расчетного метода B3LYP/6-311G(d,p) обусловлен описанным в работе [22] наилучшим согласием экспериментальных и вычисленных энергетических характеристик комплексов металлов с органическими лигандами. Методика проведения численных экспериментов была усовершенствована путем создания формализованного набора скриптов на языке Python, что обеспечило ускоренное создание входных файлов и интерпретацию выходных файлов для программы Gaussian, значительно сократило трудозатраты и время на подготовку и проведение вычислений, а также уменьшила вероятность ошибок, связанных с человеческим фактором. Содержание этой методики изложено в [23].

Расчеты строения и характеристик молекул ИДА, проделанные для вакуума, привели к следующим равновесным структурам, изображенным на рисунках 2,3.

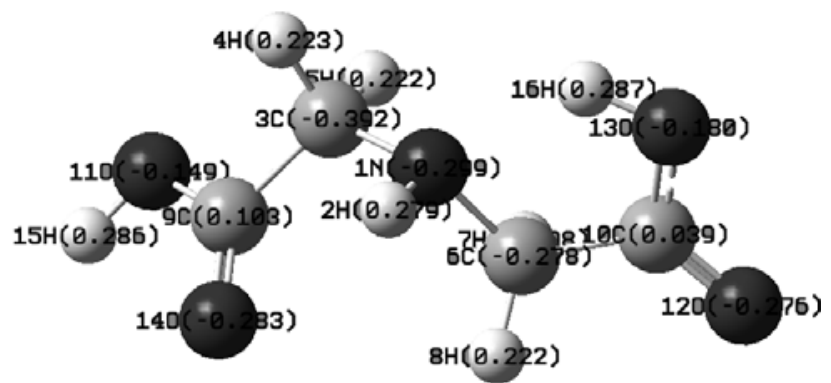

Рис. 2. Атомное строение изомера молекулы ИДА в вакууме, имеющей наименьшую энергию $(-512.475612)$.

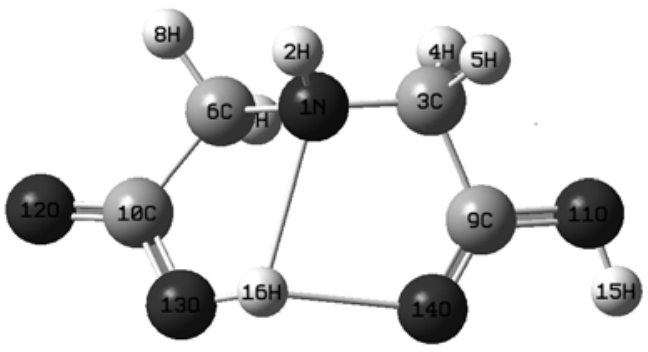

Рис. 3. Атомное строение изомера молекулы ИДА в вакууме, находящейся в возбужденном метастабильном состоянии $(-512.473170)$.

Энергии связи приведены в атомных единицах. Отметим, что в обеих молекулах протон №16 располагается вблизи иона кислорода №13 на относительно малом от него расстоянии и повернут вокруг оси СО в сторону иона азота. Ион азота и водорода имеют эффективные электрические заряды разных знаков, поэтому одной из причин этого разворота является электростатическое притяжение между этими ионами. Вторым механизмом поворота может оказаться ковалентная компонента химической связи. Для установления механизма ковалентной химической связи протона №16 были построены соответствующие молекулярные орбитали в обеих молекулах. На рисунке 4 изображены молекулярные орбитали, соединяющие ионы азота и водорода (а), водорода и кислорода (б).

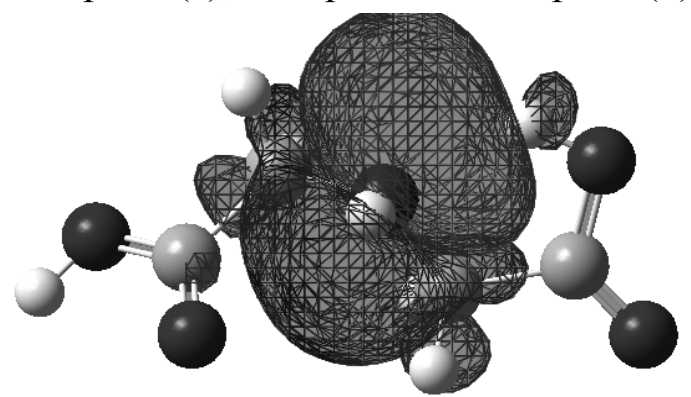

a

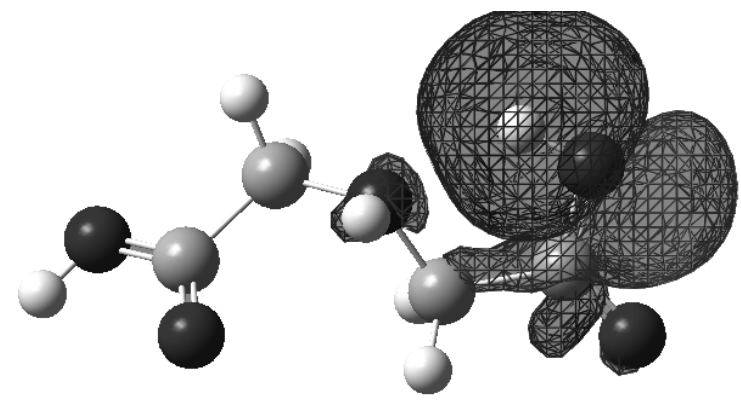

б

Рис. 4. Молекулярные орбитали стабильной молекулы молекулы ИДА, соединяющие ионы азота и кислорода (а) водорода и кислорода (б) 
Этот рисунок показывает, что в молекуле ИДА действительно имеется молекулярная орбиталь, соединяющая атом азота и водорода №16. Поэтому следует полагать, что наряду с очевидной ионной связью между этими атомами, здесь присутствует ковалентная компонента, построенная из атомных орбиталей водорода и азота. Отметим также, что на фоне доминирующей двухцентровой компоненты ковалентной связи в обоих случаях присутствуют добавки от электронных орбиталей соседних атомов, что является дополнительным упрочняющим фактором пятичленного кольца NCCOH. Результаты расчета показывают, что молекула ИДА находящаяся в стабильном состоянии в пустоте, не имеет цвиттер-структуру, так как катион водорода не захвачен ионом азота, а присоединен ионом кислорода. Аналогичные молекулярные орбитали молекулы в метастабильном состоянии представлены на рисунке 5.

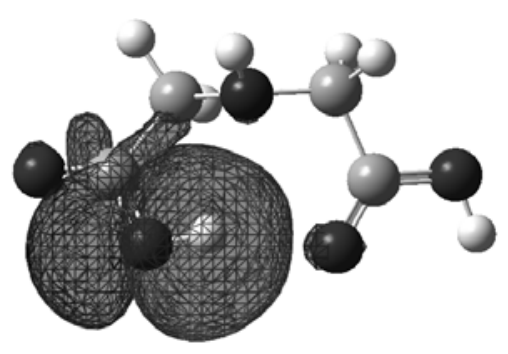

a

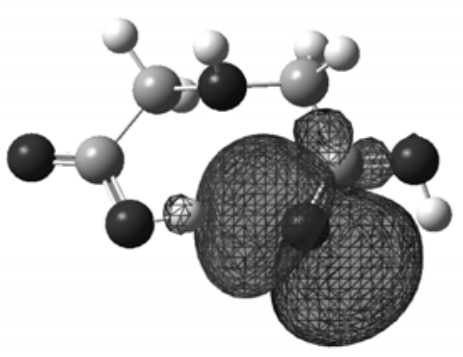

б

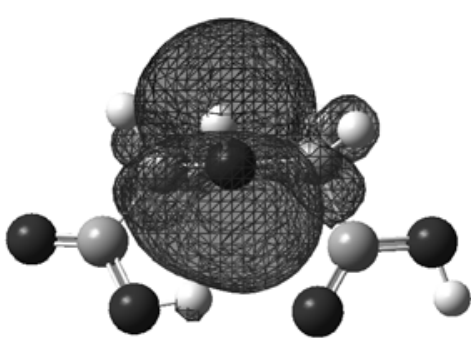

B

Рис.5. Молекулярные орбитали, связывающие протон №16 атомами кислорода $(\mathrm{a}$, б) и азота (в)

Он показывает, что протон №16 имеет ковалентные компоненты химической связи с двумя соседними атомами кислорода и атомом азота. Если учитывать эти связи, то кислотный остаток выступает для атома водорода как тридентатный лиганд. Несмотря на большее число связей атома водорода с кислотным остатком, энергия молекулы оказалась выше чем в случае двудентатной связи. Очевидно, такое увеличение обусловлено изгибом кислотного остатка вследствие сближения ионов кислорода №13 и №14. Следует отметить также, что число центров в молекулярных орбиталях молекулы основного состояния оказалось больше, чем в возбужденном изомере.

Действие водного окружения обусловлено двумя эффектами. Первым из них является индукция электрических зарядов в водной среде под действием электрических зарядов молекулы и, как следствие, индукция электрических зарядов в самой молекуле под действием наведенных электрических полей в среде. Вторым эффектом является гидратация молекулы ИДА.

Опишем первый эффект, полученный после расчетов в водной диэлектрической среде. Из сопоставления структур молекулы ИДА в вакууме и водной среде видно, что их равновесные конфигурации имеют одинаковую топологию химических связей.

Измерение длин химических связей показало, что диэлектрическая среда приводит к сокращению расстояний между атомами углерода и азота, в результате чего сжимается углеродно-азотный скелет ИДА. Результаты измерений приведены в таблице 1. Верхняя строка этой таблицы содержит номера соседних ионов, вторая строка - расстояния между ними в вакууме, нижняя - в водной среде.

В результате стрикционной деформации расстояние между крайними ионами углерода молекулы уменьшается от 4.63581 до 4.62761. Расстояния между ионами углерода и кислорода представлены в таблице 2. 
Таблица 1. Длины химических связей между ионами азота и углерода в молекуле ИДА.

\begin{tabular}{|c|c|c|}
\hline Номера соседних ионов & $\begin{array}{c}\text { Расстояния между соседними } \\
\text { ионами в вакууме }\end{array}$ & $\begin{array}{c}\text { Расстояния между соседними } \\
\text { ионами в водной среде }\end{array}$ \\
\hline $1-3$ & 1.4526 & 1.4523 \\
\hline $1-6$ & 1.4702 & 1.4616 \\
\hline $3-9$ & 1.5249 & 1.5251 \\
\hline $6-10$ & 1.5355 & 1.5269 \\
\hline
\end{tabular}

Таблица 2. Длины химических связей между ионами углерода и кислорода в молекуле ИДА.

\begin{tabular}{|c|c|c|}
\hline Номера соседних ионов & $\begin{array}{c}\text { Расстояния между соседними } \\
\text { ионами в вакууме }\end{array}$ & $\begin{array}{c}\text { Расстояния между соседними } \\
\text { ионами в водной среде }\end{array}$ \\
\hline $9-11$ & 1.3500 & 1.3379 \\
\hline $9-14$ & 1.2043 & 1.2120 \\
\hline $10-12$ & 1.2010 & 1.2136 \\
\hline $10-13$ & 1.3425 & 1.3323 \\
\hline
\end{tabular}

Из этой таблицы видно, что длины химических связей между ионами углерода и кислорода, связанных с ионами водорода, при погружении молекулы в водную среду уменьшаются, что приводит к некоторому увеличению взаимодействия между ионами кислорода и углерода. В табл. 3 представлены расстояния между ионами металлоидов и протонами. Из нее следует, что при погружении в водную среду все расстояния увеличиваются. Отметим, что наибольшее увеличение расстояния получается для протонов, располагающихся по краям молекулы.

Таблица 3. Длины химических связей между ионами металлоидов и протонами в молекуле ИДА.

\begin{tabular}{|c|c|c|}
\hline Номера соседних ионов & $\begin{array}{c}\text { Расстояния между соседними } \\
\text { ионами в вакууме }\end{array}$ & $\begin{array}{c}\text { Расстояния между соседними } \\
\text { ионами в водной среде }\end{array}$ \\
\hline $1-2$ & 1.0155 & 1.0259 \\
\hline $3-4$ & 1.0954 & 1.0962 \\
\hline $3-5$ & 1.0927 & 1.0945 \\
\hline $6-7$ & 1.0940 & 1.0961 \\
\hline $6-8$ & 1.0926 & 1.0978 \\
\hline $11-15$ & 0.9698 & 0.9938 \\
\hline $13-16$ & 0.9811 & 0.9916 \\
\hline
\end{tabular}

Далее представляются результаты вычислений валентных углов для молекулы ИДА в вакууме и водной среде. В таблицах 4-6 представлены валентные углы между ионами соседних ионов кислорода, углерода и азота.

Таблица 4. Валентные углы между соседними ионами кислорода

\begin{tabular}{|c|c|c|}
\hline Номера соседних ионов & $\begin{array}{c}\text { Валентные углы между со- } \\
\text { седними ионами в вакууме }\end{array}$ & $\begin{array}{c}\text { Валентные углы между со- } \\
\text { седними ионами в водной } \\
\text { среде }\end{array}$ \\
\hline $9,3,1$ & 114.3064 & 115.4203 \\
\hline $3,1,6$ & 116.0765 & 116.2408 \\
\hline $1,6,10$ & 111.6755 & 111.2866 \\
\hline
\end{tabular}

Далее представлены изменение двугранных углов между плоскостями, определяемыми последовательностью номеров элементов, входящих в состав молекулы. 
Таблица 5. Валентные углы между соседними ионами углерода

\begin{tabular}{|c|c|c|}
\hline Номера соседних ионов & $\begin{array}{c}\text { Валентные углы между со- } \\
\text { седними ионами в вакууме }\end{array}$ & $\begin{array}{c}\text { Валентные углы между со- } \\
\text { седними ионами в водной } \\
\text { среде }\end{array}$ \\
\hline $3,9,11$ & 111.3893 & 111.1772 \\
\hline $3,9,14$ & 124.9454 & 124.9120 \\
\hline $6,10,12$ & 122.6885 & 123.2224 \\
\hline $6,10,13$ & 114.1094 & 115.1250 \\
\hline
\end{tabular}

Таблица 6. Валентные углы между соседними ионами азота

\begin{tabular}{|c|c|c|}
\hline Номера соседних ионов & $\begin{array}{c}\text { Валентные углы между со- } \\
\text { седними ионами в вакууме }\end{array}$ & $\begin{array}{c}\text { Валентные углы между со- } \\
\text { седними ионами в водной } \\
\text { среде }\end{array}$ \\
\hline $9,11,15$ & 107.9032 & 109.3927 \\
\hline $10,13,16$ & 105.6215 & 106.8840 \\
\hline $10,9,14$ & 123.6501 & 123.8990 \\
\hline $12,10,13$ & 123.1875 & 121.6461 \\
\hline
\end{tabular}

Таблица 7. Двугранные углы между плоскостями

\begin{tabular}{|c|c|c|}
\hline $\begin{array}{c}\text { Номера элементов, } \\
\text { входящих в двугранный угол }\end{array}$ & $\begin{array}{c}\text { Двугранный угол } \\
\text { между плоскостями в вакуу- } \\
\text { ме }\end{array}$ & $\begin{array}{c}\text { Двугранный угол } \\
\text { между плоскостями в водной } \\
\text { среде }\end{array}$ \\
\hline $9,3,1,6$ & 71.2811 & 70.1069 \\
\hline $3,1,6,10$ & 141.6031 & 139.4157 \\
\hline $11,9,3,1$ & 170.6256 & 171.3896 \\
\hline $14,9,3,1$ & 10.7504 & 9.8187 \\
\hline $1,6,10,12$ & 170.1656 & 171.4780 \\
\hline $1,6,10,13$ & 11.1817 & 9.4379 \\
\hline $15,11,9,14$ & 0.6787 & 0.8904 \\
\hline $16,13,10,12$ & 179.2583 & 179.2598 \\
\hline $1,16,13,10$ & 6.2780 & 5.3012 \\
\hline
\end{tabular}

Результаты вычислений геометрических характеристик молекулы ИДА в вакууме и в водной среде показывают, что строение молекул сохраняется. Изменение длин валентных связей и валентных углов составляет примерно проценты от исходных. Несколько большее изменение происходит в двугранных углах.

Изменение зарядовых состояний ионов после помещения молекулы ИДА в водную среду представлено на рисунке 6.

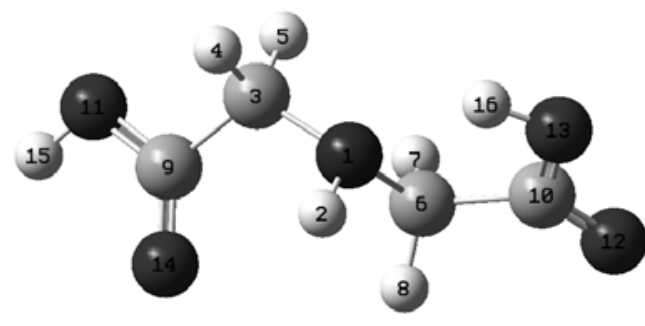

a

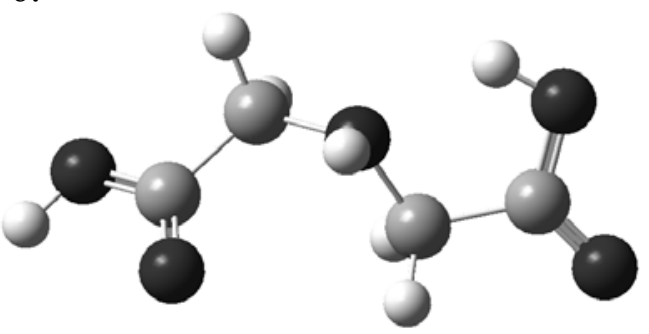

6

Рис. 6. Строение молекулы ИДА в вакууме (а) и водной среде (б)

Сравнение эффективных зарядов показывает, что погружение молекулы ИДА в водную среду увеличивает по модулю заряды всех ионов. Механизмом этого эффекта является появление зарядов противоположного знака вблизи каждого иона. Этот индуцированный заряд приводит к дополнительному притяжению зарядов ио- 
нов, что и приводит к увеличению суммарного эффективного заряда. Особенно сильно, как это видно на рисунке 7, проявляется этот эффект для крайних ионов кислорода, которые находятся в непосредственно контакте с диэлектрической средой и испытывающих прямое воздействие с ее стороны. Ионы, расположенные более глубоко в молекуле ИДА, слабее подвержены прямому воздействию среды, но, находясь в контакте с внешними ионами, испытывают индуцированное увеличение заряда. Этим эффектом можно объяснить относительно небольшое изменение величины заряда этих ионов. Относительно малое изменение эффективного заряда протонов следует из сравнительно малой поляризуемости ионов водорода.

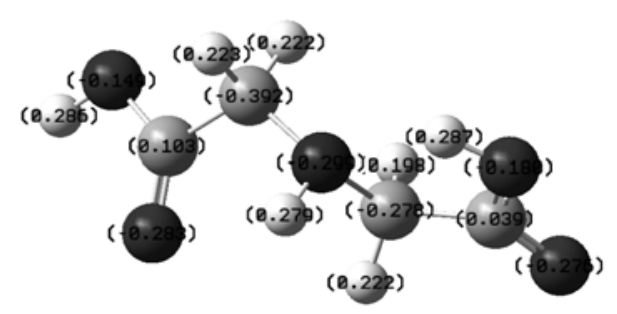

a

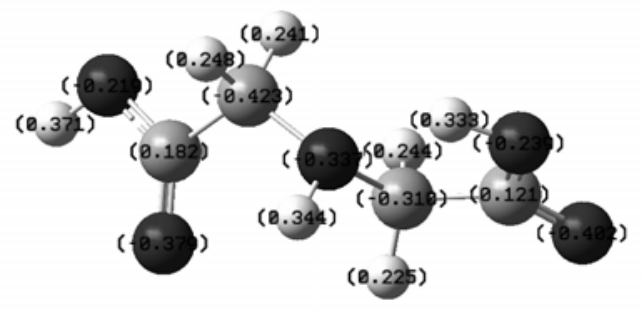

6

Рис. 7. Распределение эффективных электрических зарядов ионов молекулы ИДА в вакууме (а) и в водной среде (б).

Одночастичные орбитали ИДА в водной среде.
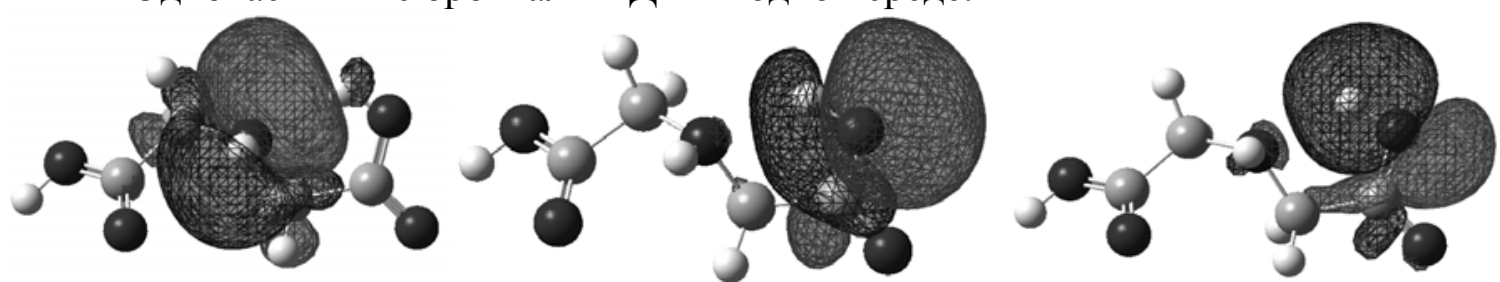

Рис. 8. Молекулярные орбитали, связывающие протон №16 атомами кислорода $(\mathrm{a}$, б) и азота (в)

Сравнение орбиталей, представленных на рисунках 5 и 8 показывает, что действие диэлектрической среды приводит к появлению дополнительной орбитали, участвующих в ковалентной связи между ионами азота и водорода. Раскрытие файла, содержащего параметры волновых функций, показало, что они становятся более широкими, что и приводит к появлению более сильного перекрывания разных атомных волновых функций. В заключение можно сделать вывод о том, что совокупность рассмотренных эффектов, связанных с поляризацией водной среды, приводит к увеличению энергии связи молекулы ИДА от 512,475612 до 512,510362 и ее стабильности. Однако, ион водорода №16 располагается вблизи иона кислорода №13, а его электронный заряд частично переносится на этот анион. Таким образом, учет влияния водного окружения как диэлектрической среды само по себе не приводит к стабилизации цвиттер-ионной структуры молекулы ИДА.

Цвиттер-структура. Экспериментально установлено, что в водных растворах молекула ИДА имеет цвиттер-ионную структуру. В настоящем разделе ставится задача проведения расчетов с целью установления строения и условия стабильного существования этой структуры.

Для дополнительного подтверждения этого вывода были проведены вычисления строения молекулы ИДА, в предположении, что исходной структурой молекулы является цвиттер-структура, в которой ион водорода №16 расположен вблизи к иону азота, так что между ними образуется ковалентная связь. В результате получается молекула, изображенная на рисунке 9. 


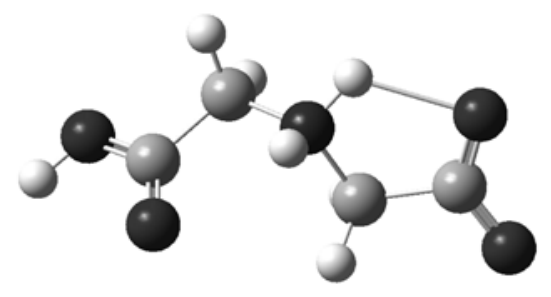

Рис. 9. Строение цвиттер- молекулы ИДА

На этом рисунке длинная линия, соединяющая ионы водорода и кислорода обозначает смещение иона водорода от иона кислорода к иону азота. Применение вычислительной процедуры с оптимизацией структуры такой молекулы в вакууме показало, что ион водорода №16 вернулся в позицию вблизи иона кислорода №13. Таким образом, в условиях вакуума равновесная цвиттер-структура молекулы ИДА отсутствует. Учет диэлектрической среды привел к появлению механически равновесной цвиттер-структуры молекулы ИДА. Однако энергия этой молекулы 512.508855 оказалась выше энергии основного состояния молекулы ИДА, равной 512.510362 , поэтому это состояние молекулы следует считать возбужденным метастабильным изомером ИДА. Отсюда следует, что учет диэлектрической среды не приводит к появлению равновесной цвиттер-ионной структуре молекулы, но способствует ее возникновению. Физическая причина последнего эффекта лежит в неоднородности дополнительной поляризации ионов молекулы ИДА. Из рисунка 7 видно, что дополнительный эффективный заряд, обусловленный поляризационным эффектом, для ионов кислорода в несколько раз превосходит аналогичный заряд иона азота. Этот заряд способствует выталкиванию положительно заряженного иона водорода в область относительно малого заряда около иона азота.

Далее было исследовано влияние эффекта гидратации на стабилизацию цвиттер-структуры молекулы ИДА. В качестве простейшей модели этого эффекта были рассмотрены молекула ИДА в основном и цвиттер-состоянии плюс одна молекула воды, расположенная в активной области молекулы ИДА. Результаты расчета представлены на рис. 10.

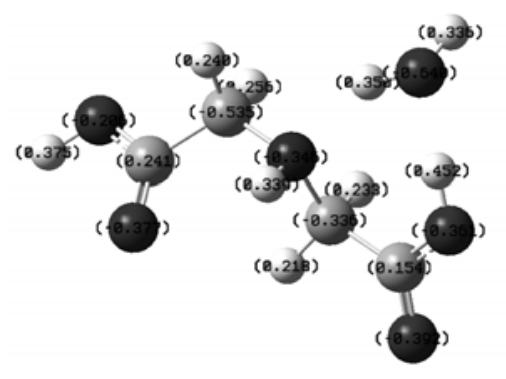

a

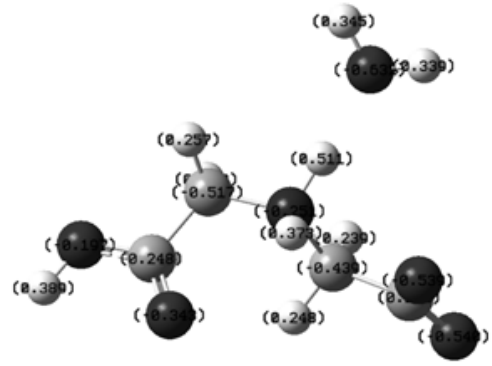

б

Рис.10. Строение гидрата (а) и цвиттер- гидрата ИДА(Н $\left.{ }_{2} 0\right)$ (б)

Вычисленные энергии гидратов соответственно равны $-558, .83833$ и -558.987579. Отсюда следует, цвиттер-гидрат становится основным состоянием молекулы. Таким образом, учет одной молекулы воды приводит к стабилизации цвиттер структуры. Поэтому можно ожидать, что увеличение числа присоединенных молекул воды, которое реализуется в водной среде, приведет к еще большей стабилизации этой структуры. Отметим, что в результате присоединения молекулы воды в двух изомерах ИДА оказываются существенно разными суммарные заряды азотноводородных фрагментов. Как видно на рисунке 10, в левом изомере заряд этого фрагмента близок к нулю, в то время как цвиттер-изомере он равен +0.633 , что по- 
рождает относительно большую энергию притяжения иона кислорода, принадлежащего молекуле воды, и является стабилизирующим фактором для этой структуры молекулы.

\section{Обсуждение результатов}

Для установления характера химической связи между молекулами ИДА и воды был проведен орбитальный анализ гидратированных молекул.

На рис. 11 представлены орбитали, соединяющие молекулу воды с молекулой ИДА в конфигурации, соответствующей основному состоянию.
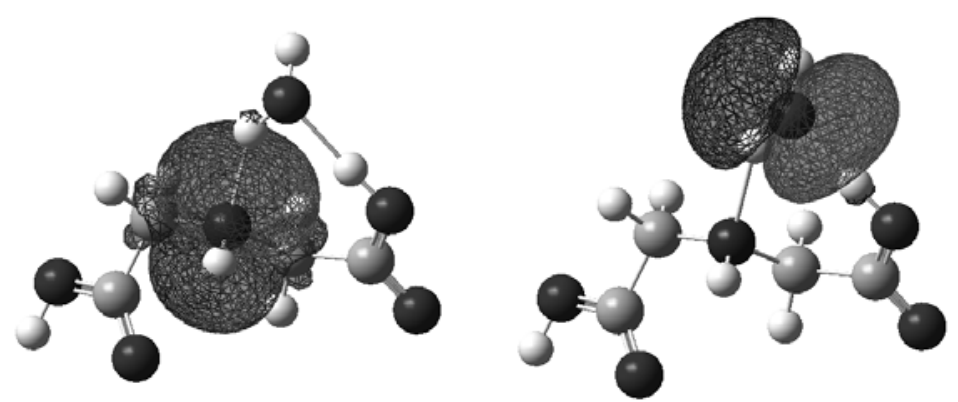

Рис. 11. Двуцентровые орбитали, связывающие молекулы воды и ИДА в стабильном состоянии

Отсюда видно, что молекула воды удерживается около молекулы ИДА двумя орбиталями, соединяющими разные пары водород-азот и водород кислород. Молекулярные орбитали, соединяющие цвиттер-молекулу ИДА и молекулу воды изображены на рис.12.
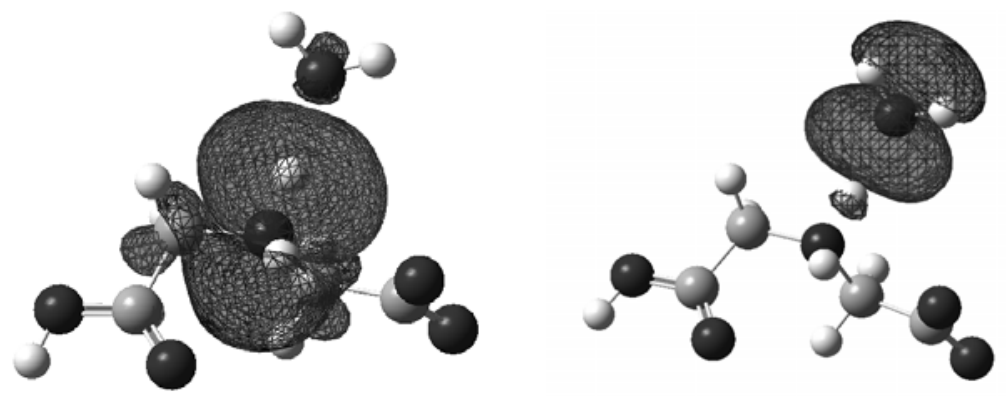

Рис. 12. Орбитали, связывающие молекулы воды и ИДА в цвиттер состоянии

Как видно из этого рисунка, связывание молекулы воды происходит двумя орбиталями, имеющими общий ион водорода. Одна из орбиталей является трехцентровой и соединяет ионы азота, водорода и кислорода. Возможно, появление такой орбитали является доминирующим физико-химическим механизмом стабилизации цвиттер-структуры молекулы ИДА в водной среде.

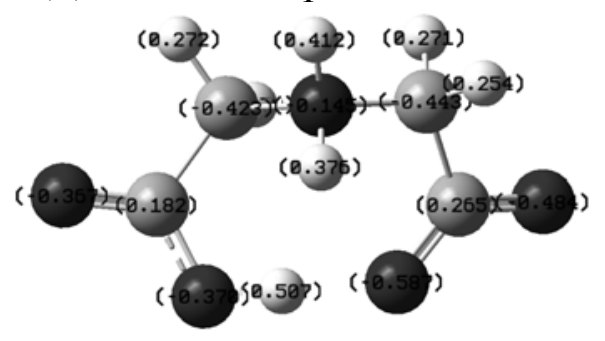

Рису. 13. Цвиттер-структура молекулы ИДА с энергией -512.501710 
Другой изомер ИДА, имеющий цвиттер-структуру получается из молекулы, представленной на рис. 2, путем смещения иона водорода №16 к иону азота и двух поворотов примерно на $180^{\circ}$ вокруг осей 3-9 и 9-11. В результате получается молекула, изображенная на рис. 13.

Отметим, водородно-азотный фрагмент, как и в случае, рассмотренном выше, имеет относительно большой положительный заряд, равный 0,632. Энергия возбуждения этого изомера, полученная с учетом поляризуемости среды, но без учета гидратации, составляет 0.0087. Присоединение одной молекулы воды приводит к гидрату ИДА, представленному на рисунке 14.

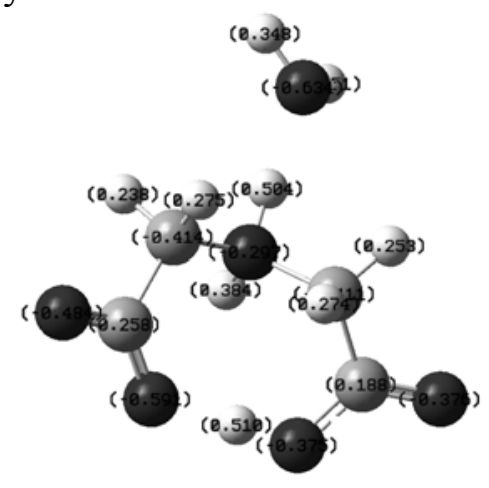

Рис. 14. Гидрат молекулы ИДА в цвиттер-ионном состоянии с энергией $-558.987579$

Разность энергий между энергиями гидратов в основном и возбужденном цвиттер-ионных состояниях составила 0.0019 , что примерно в четыре раза меньше аналогичной величины, полученной без учета гидратации. Этот результат также свидетельствует об определяющей роли гидратации в формировании цвиттер-ионов в водных растворах.

\section{Заключение}

В заключение сформулируем основные результаты проделанных вычислений. Молекула ИДА имеет несколько изомеров, полученных вращением молекулярных групп вокруг химических связей C-C, C-N, C-O. Если молекула ИДА находится в вакууме, то вблизи иона азота находится только один протон. Помещение молекулы ИДА в водную среду приводит к появлению цвиттер-структуры, в которой около иона азота локализуются два протона. Однако этот изотоп имеет повышенную энергию по отношению к энергии основного состояния, поэтому его следует рассматривать как возбужденное состояние молекулы ИДА. Таким образом, учет водного окружения как диэлектрической среды не приводит к появлению стабильной цвиттермолекулы ИДА.

Гидратация молекулы ИДА одной молекулой воды приводит к отрыву протона, химически связанного с одним из атомов кислорода, и присоединение его к иону азота. Электронным механизмом этого переноса является образование двух низкоэнергетических орбиталей, одна из которых имеет структуру трехцентровой орбитали, связывающая ионы азота, кислорода воды и соединяющего их протона.

Таким образом, можно сделать вывод, что причиной появления цвиттерструктуры молекулы ИДА в водном растворе является присутствие ковалентной компоненты химической связи, которая возникает в водном растворе при гидратация молекул ИДА. 


\section{Список литературы}

1. Schwarzenbach G., Flashka H. Die komplexometrische Titration. Stuttgart.1965. $360 \mathrm{p}$.

2. Inczedy J. Analitical applications of complex equilibria. Budapest. 1976. 376 p.

3. Архипова О.Г., Зорина Л.А., Сорокина Н.С. Комплексоны в клинике профессиональных болезней. М. Медицина. 1975. $160 \mathrm{c}$.

4. Семенов Д.И., Трегубенко И.П. Комплексоны в биологии и медицине. Свердловск. УНЦ АН СССР. 1984. 280 с.

5. Кельциева О.А., Гладилович В.Д., Подольская Е.П. // Научное приборостроение. 2013. Т. 23. № 1. С. 74-85.

6. Дятлова Н.М., Ластовский Р.П. // Успехи химии. 1965. Т. 34. № 7. С. 1153-1184.

7. Hartley F. R. Supported metal complexes. A new generation of catalysts. Dordrecht etc, D. Reidel Publishing, 1985. 360 p.

8. Тертых В.А., Белякова Л.А. Химические реакции с участием поверхности кремнезема. Киев. Наукова думка. 1991. 262 c.

9. Kondo K., Sumi H., Matsumo M. // Separation Science and Technology. 1996. Vol. 31. No 12. pp.1771-1775.

10..Fortes M.C.B, Martins A.H., Benedetto J.S. // Minerals Engineering. 2003. No 16. pp. 659-663.

11.Liu D., Cant N.W., Smith A.J., Wainwright M.S. // Appl. Catalysis A: General. 2006. Vol. 297. pp. 18-23.

12.Tian J., Shi H., Li X., Yin Y., Chen L. //
Green Chemistry. 2012. Vol. 14. pp. 1990-2000.

13.Feng L., Chen J., Ren B. // Agrochemistry. 2006. Vol. 45. pp. 12-14.

14.Лисичкин Г.В. Модифицированные кремнеземы в сорбции, катализе и хроматографии. М. Химия. 1986. 248 с.

15.Лисичкин Г.В. // Соровский образовательный журнал. 1996. № 4. С. 5359.

16.Schwarzenbach G., Kampitsch E., Steiner R. // Helvetica chimica acta. 1945. Vol. 28. No 1. pp. 828-840.

17.Schwarzenbach G., Biedermann W. // Helvetica chimica acta. 1948. Vol. 31. No 2. pp. 331-340.

18.Biedermann W., Schwarzenbach G. // Chimia, 1948. No 2. pp 56-58.

19. Nakamoto K. Infrared and Raman spectra of inorganic and coordination compounds. New York etc. A Wiley Interscience Publication. $1986.536 \mathrm{p}$.

20.Дятлова Н.М., Темкина В.Я., Попов К.И. Комплексоны и комплексонаты металлов. Москва, Химия, 1988, 544 с.

21.Школьникова Л.М., Парай-Кошиц М.А., Дятлова Н.М. Проблемы кристаллохимии. M. 1986. C. 32-87.

22.Antony J., Hansen B., Hemmingsen L., Bauer R. // J. Phys. Chem. A. 2000. Vol. 104. pp. 6047-6055.

23.Semenov A.M. // X Международная конференция молодых учёных по химии «Менделеев-2017». Санкт-Петербург. 2017. C. 385 .

\section{References}

1. Schwarzenbach G. Flashka H. Die komplexometrische Titration. Stuttgart, 1965, $360 \mathrm{p}$.

2. Inczedy J. Analitical applications of complex equilibria. Budapest, 1976, 376 p.

3. Arkhipova O.G., Zorina L.A., Sorokina N.S. Kompleksony v klinike professional'nykh boleznei. M., Meditsina Publ., 1975, 160 p.

4. Semenov D.I., Tregubenko I.P. Kompleksony $\mathrm{v}$ biologii $\mathrm{i}$ meditsine. Sverdlovsk, USC AS USSR, 1984, 280 p.

5. Kel'tsieva O.A., Gladilovich V.D., Podol'skaya E.P., Nauchnoe priborostroenie, 2013, Vol. 23, No 1, pp. 74-85.

6. Dyatlova N.M., Lastovskii R.P., Uspekhi khimii, 1965, Vol. 34, No 7, pp. 1153-1184.
7. Hartley F. R. Supported metal complexes. A new generation of catalysts. Dordrecht etc, D. Reidel Publishing, 1985, $360 \mathrm{p}$.

8. Tertykh V.A., Belyakova L.A. Khimicheskie reaktsii s uchastiem poverkhnosti kremnezema. Kiev, Naukova dumka, 1991, 262 p.

9. Kondo K., Sumi H., Matsumo M., Separation Science and Technology, 1996, Vol. 31, No 12, pp. 1771-1775.

10.Fortes M.C.B, Martins A.H., Benedetto J.S., Minerals Engineering, 2003, No 16, pp. 659-663.

11.Liu D., Cant N.W., Smith A.J., Wainwright M.S., Appl. Catalysis A: General, 2006, Vol. 297, pp. 18-23. 
12.Tian J., Shi H., Li X., Yin Y., Chen L., Green Chemistry, 2012, Vol. 14, pp. 1990-2000.

13.Feng L., Chen J., Ren B., Agrochemistry, 2006, Vol. 45, pp. 12-14.

14.Lisichkin G.V. Modifitsirovannye kremnezemy $\mathrm{v}$ sorbtsii, katalize i khromatografii. Moskow, Khimiya Publ., 1986, 248 p.

15.Lisichkin G.V., Sorovskii obrazovatel'nyi zhurnal, 1996, No 4, pp.53-59.

16. Helvetica chimica acta. 1945. Vol. 28. No 1. pp. 828-840.

17.Schwarzenbach G., Biedermann W., Helvetica chimica acta, 1948. Vol. 31. No 2. pp. 331-340.

18.Biedermann W., Schwarzenbach G., Chimia, 1948, No 2, pp 56-58.

Даринский Борис Михайлович - д.ф.-м.н., профессор кафедры материаловедения и индустрии наносистем химического факультета Воронежского государственного университета, Воронеж

Петрушенко Руслан Викторович - магистрант кафедры материаловедения и индустрии наносистем химического факультета Воронежского государственного университета, Воронеж

Селеменев Владимир Федорович - д.Х.н., профессор, зав. кафедрой аналитической химии химического факультета Воронежского государственного университета, Воронеж
19. Nakamoto K. Infrared and Raman spectra of inorganic and coordination compounds. New York etc, A Wiley Interscience Publication, 1986, $536 \mathrm{p}$.

20.Dyatlova N.M., Temkina V.Ya., Popov K.I. Kompleksony i kompleksonaty metallov. Moskow, Khimiya Publ., 1988, 544 p.

21.Shkol'nikova L.M., Parai-Koshits M.A., Dyatlova N.M., Problemy kristallokhimii, M., 1986, pp. 32-87.

22.Antony J., Hansen B., Hemmingsen L., Bauer R., J. Phys. Chem. A, 2000, Vol. 104, pp. 6047-6055.

23.Semenov A.M., $X$ Mezhdunarodnaya konferentsiya molodykh uchenykh po khimii «Mendeleev-2017», April 4-7, 2017, St.Peterburg, 2017, p.385

Darinskii Boris M. - doctor of physical and mathematical sciences, professor of the department of Material Science and Industry of Nanosystems, chemical faculty, Voronezh State University, Voronezh, e-mail: darinskii@mail.ru

Petrushenko Ruslan V. - student of the department of Material Science and Industry of Nanosystems, chemical faculty, Voronezh State University, Voronezh

Selemenev Vladimir F. - doctor of chemical sciences, professor, head of the department of Analytical Chemistry, chemical faculty, Voronezh State University, Voronezh 